\title{
FACTORES ASOCIADOS A LA PARTICIPACIÓN LABORAL DE TRABAJADORES SECUNDARIOS DE LOS HOGARES DEL DISTRITO DE GUADALUPE
}

\section{FACTORS ASSOCIATED TO THE LABOR PARTICIPATION OF SECONDARY WORKERS OF THE HOMES OF THE DISTRICT OF GUADALUPE}

\section{RESUMEN}

\author{
Carlos Sánchez ${ }^{a}$, Carlos Minchón ${ }^{b}$
}

\begin{abstract}
El presente trabajo fue realizado con el propósito de determinar los factores asociados a la participación laboral de trabajadores secundarios de los hogares del distrito de Guadalupe, provincia de Pacasmayo, departamento de La Libertad en Perú. Se evaluó factores del jefe del hogar (ingreso económico, educación, sexo y edad), del trabajador secundario (edad, años de estudio, parentesco con el jefe y sexo) y de la familia (ingreso familiar, tamaño de la familia, presencia de miembros en la familia menores a 5 años y de 5 a 13 años). Como indicadores de la participación laboral se consideró a la decisión de participar en el mercado laboral del trabajador secundario como al número de horas semanales laboradas.

La muestra obtenida mediante muestreo unietápico, estuvo conformada por 552 viviendas (UPM's) y 1094 trabajadores secundarios (USM's), recolectándose la información mediante encuesta, entre enero y febrero del 2008. Se empleó el modelo de regresión probit y el modelo de regresión tobit, estimándose los modelos con Stata 9.2. Los factores asociados positivamente fueron la edad del trabajador secundario, los ingresos familiares. Los factores asociados negativamente fueron el ingreso del jefe del hogar y el tamaño de la familia. Los factores cualitativos fueron jefe del hogar mujer, presencia de menores de 5 años en la familia y trabajador secundario varón.
\end{abstract}

Palabras claves: Trabajador secundario, participación laboral, factores asociados, probit, tobit.

\section{ABSTRACT}

The present work was carried out in order to determine the factors associated with the labour participation of secondary workers of the homes in Guadalupe, Pacasmayo, Peru. Several factors were evaluated head of home (salary, education, sex and age), of the secondary worker (age, years of study, kinship with the head and sex), and of the family (salary family, family size, family members under 5 years and from 5 to 13 years old 5 years and of 5 to 13 years).Indicators of the labour participation it was considered the decision to participate to the labour market as to the number of weekly labour hours. The sample, obtained the through the unietapico sample, was conformed by 552 houses and 1094 secondary workers, collecting the information by surveys, between January and February 2008. The Probit and Tobit models regression were used but it was esteemed the models with Stata 9.2. The positive associated factors were the age of the secondary worker, the familiar income. The negative associated factors were the head of the homes salary the family size. The qualitative factors were head of the home woman, formed members under 5 years old and male secondary workes.

Key words: Secondary worker, labour participation, associated factors, probit, tobit.

\section{INTRODUCCIÓN}

El comportamiento de la oferta de trabajo de las cónyuges ante el desempleo de los jefes de hogar varones es un tema que ha recibido mucha atención en economía laboral, incorporándose en un estudio realizado en Argentina a otros miembros del hogar (hijos y otros miembros) aparte de las cónyuges en condición de reserva laboral -denominados trabajadores secundarios (cónyuges, hijos dependientes y personas mayores de 65 años no jefes del hogar)- quienes se incorporan al mercado laboral ante la necesidad de aportar para cubrir el presupuesto familiar ${ }^{1}$. Otro aspecto incluido en este trabajo fue la situación ocupacional del principal aportante del hogar, donde no solamente el desempleo sino también su reinserción en puestos de baja remuneración relativa o de escasa intensidad pueden incidir en la decisión de los trabajadores secundarios de participar en el mercado laboral (modelo logit), y de la intensidad con la que lo hacen (modelo tobit). El estudio fue realizado para los años 1992, 1995 y 1999, por separado, analizando la situación ocupacional del jefe de familia como variable independiente en dos maneras. La primera comparando los jefes con ocupación plena frente a los que no tienen ocupación plena, y la segunda comparando las modalidades de ocupación no plena (desocupado, subocupado o informal) frente a la ocupación plena del jefe. El estudio también incluyó como variables independientes a características individuales del trabajador secundario y a características de la familia.

En el Perú se ha venido incrementando la tasa de participación laboral, específicamente de las mujeres. La realidad peruana lleva a proponer que la decisión de participar en el mercado de trabajo, además de la variables individuales, de las características de la familia, tal como fue analizado en un estudio basado en la ENAHO 1998-III, correspondiente a Perú urbano ${ }^{2}$. Se examinó tanto la decisión de participar en el mercado laboral como de la tasa de participación, empleando según se indica un modelo tipo logit en el primer caso y el modelo de regresión lineal en el segundo. Por la ecuación mostrada en el modelo tipo logit, la prueba t indicada en los resultados y el programa SPSS

${ }^{a, b}$ Departamento de Estadística, Facultad de Ciencias Físicas y Matemáticas, Universidad Nacional de Trujillo, Perú. 
empleado, consideramos que en realidad aplicaron el modelo lineal de probabilidad. La tasa de participación está dada por la proporción de los miembros de la familia en edad de trabajar que son miembros de la población económicamente activa (PEA). Otra particularidad del estudio es que elaboraron modelos separados para hogares promedio y por género; para hogares nucleares, extendidos o compuestos; y para una combinación de ambas variables.

Prácticamente este estudio fue actualizado en Perú, comparando hogares cuyo jefe del hogar es un trabajador independiente frente a los de trabajadores independientes, basado en la ENAHO 2001-III. En el caso de la decisión del trabajador secundario de participar o no en el mercado laboral el modelo empleado fue el logit, sin embargo aún cuando se pretendió incluir como variable independiente a la ocupación del trabajador principal (independientedependiente), su rol en el modelo no es claro.

Los mejores argumentos teóricos para abordar este tema fueron exhibidos en el reiterado uso de la ENAHO 2001-III, cuando se utilizó el modelo probit para estudiar la decisión del trabajador secundario de incorporarse a la fuerza laboral, así como el modelo tobit para estudiar la intensidad con que decide incorporarse a este mercado (medida en el número de horas semanales) ${ }^{4}$. La propuesta de incluir estos modelos y más variables independientes debe ser considerada. Por otro lado, la ENAHO por ser una encuesta demasiado amplia podría presentar limitaciones en cuanto a calidad de información se refiere. Esto presupone que los modelos en referencia deben ser estimados empleando información de fuentes primarias.

El presente estudio tiene el propósito de determinar los factores asociados a la participación laboral de trabajadores secundarios - decisión de incorporarse al mercado laboral y de la intensidad que lo hace- del distrito de Guadalupe.

\section{METODOLOGÍA}

El estudio fue realizado en los trabajadores secundarios del distrito de Guadalupe, provincia de Pacasmayo, departamento de La Libertad, en Perú, entre enero y febrero del 2008.

Se evaluó factores del jefe del hogar (ingreso económico, educación, sexo y edad), del trabajador secundario (edad, años de estudio, parentesco con el jefe y sexo) y de la familia (ingreso familiar, tamaño de la familia, presencia de miembros en la familia menores a 5 años y de 5 a 13 años). Como indicadores de la participación laboral se consideró a la decisión de participar en el mercado laboral del trabajador secundario como al número de horas semanales laboradas.

El marco muestral estuvo conformado por las 6867 viviendas registradas en el plano catastral del distrito de Guadalupe, actualizado a agosto del 2006, proporcionado por el Municipio de Guadalupe.

La muestra fue obtenida mediante muestreo unietápico. Las unidades primarias de muestreo (UPM) fueron las viviendas y las unidades secundarias de muestreo (USM) los trabajadores secundarios. La muestra estuvo conformada por 552 viviendas y 1094 trabajadores secundarios. El número de viviendas que conformaron las UPM's de la muestra fueron determinadas asumiendo máxima variabilidad, confianza del 95\% y precisión del $4 \%$. En cambio, se consideró todos los trabajadores secundarios de las UPM's seleccionadas.

El diseño de investigación corresponde a un diseño no experimental, transversal y causal, cuyo esquema es:

Factores

- Trabajador principal

- Trabajador secundario

- Familia

La decisión del trabajador secundario participar en el mercado laboral fue modelado mediante el modelo de regresión probit. El número de horas semanales laboradas por el trabajador secundario fue modelado a través del modelo probit. Las variables independientes fueron codificadas como sigue:

\section{CODIFICACIÓN DE FACTORES}

\begin{tabular}{|c|c|c|c|}
\hline Factores & Denominación & Categoría & Código \\
\hline $\begin{array}{c}\text { Sexo del Jefe de } \\
\text { hogar(Referencia : Femenino) }\end{array}$ & SJ & $\begin{array}{l}\text { Masculino } \\
\text { Femenino }\end{array}$ & $\begin{array}{l}1 \\
0\end{array}$ \\
\hline \multirow[b]{2}{*}{$\begin{array}{c}\text { Parentesco } \\
\text { (Referencia: Otro Pariente) }\end{array}$} & $\mathrm{H}$ & $\begin{array}{l}\text { Trab. Secund. Hijo } \\
\text { Otro }\end{array}$ & $\begin{array}{l}1 \\
0 \\
\end{array}$ \\
\hline & $\mathrm{C}$ & $\begin{array}{l}\text { Trab. Secund. } \\
\text { Cónyuge } \\
\text { Otro }\end{array}$ & $\begin{array}{l}1 \\
0\end{array}$ \\
\hline $\begin{array}{l}\text { Integrantes menores a } 5 \\
\text { años. } \\
\text { (Referencia: Otro integrante) }\end{array}$ & $\mathrm{M}_{1}$ & $\begin{array}{c}\text { Hogares con } \\
\text { miembros menores } \\
5 \text { años } \\
\text { Otro }\end{array}$ & $\begin{array}{l}1 \\
0\end{array}$ \\
\hline $\begin{array}{l}\text { Integrantes entre } 5 \text { y } 13 \\
\text { años. } \\
\text { (Referencia: Otro integrante) }\end{array}$ & $\mathrm{M}_{2}$ & $\begin{array}{c}\text { Hogares con } \\
\text { miembros entre } 5 \text { y } \\
13 \text { años } \\
\text { Otro }\end{array}$ & $\begin{array}{l}1 \\
0\end{array}$ \\
\hline $\begin{array}{c}\text { Sexo del trabajador } \\
\text { secundario(Referencia: } \\
\text { Femenino) }\end{array}$ & ST & $\begin{array}{l}\text { Masculino } \\
\text { Femenino }\end{array}$ & $\begin{array}{l}1 \\
0\end{array}$ \\
\hline Ingreso del Jefe de Hogar & $\mathrm{IJ}$ & & \\
\hline Educación del jefe & EJ & & \\
\hline Edad del jefe & DJ & & \\
\hline $\begin{array}{l}\text { Edad Trabajador } \\
\text { Secundario }\end{array}$ & ES & & \\
\hline Años de estudio & $\mathrm{AE}$ & & \\
\hline Ingresos Familiares & IF & & \\
\hline Tamaño familiar & TF & & \\
\hline
\end{tabular}

Los modelos probit y tobit fueron estimados empleando Stata, versión 9.2. En el caso del modelo probit se evalúa además el efecto marginal de cada variable independiente, el cual permite comparar el efecto de una variable con otra, así como determinar la magnitud del efecto que produce en la decisión de laborar del trabajador secundario el correspondiente cambio en cada una de las variables independientes [5].

\section{RESULTADOS Y DISCUSIÓN}

En el distrito de Guadalupe, la muestra estuvo comprendida por 1094 trabajadores secundarios que conformaron la fuerza laboral de reserva, de los cuales $739(67.6 \%)$ no tomaron la decisión de trabajar, es decir 
ni se encontraban laborando ni estuvieron buscando trabajo durante el periodo de estudio. A nivel de Perú urbano, al III trimestre del 2001, Abanto ${ }^{4}$ determinó que el $43.9 \%$ de los trabajadores no tomó la decisión de laborar.

\section{- La deCISIÓn de participar en mERCAdo LABORAL}

El análisis de los factores asociados a la participación laboral de los trabajadores incluyó los factores del jefe del hogar, del trabajador secundario y de la familia, como se indica en la metodología. Sin embargo, no todos fueron significativos.

El modelo de regresión probit para la decisión de laborar del trabajador secundario es mostrado en la tabla 1, el cual presenta los coeficientes de regresión estimado después de eliminar los factores no significativos corriendo nuevamente el programa Stata.

\section{TABLA N ${ }^{\circ} 1$ \\ ANÁLISIS PROBIT DE LA PARTICIPACIÓN LABORAL RETIRANDO LOS FACTORES NO SIGNIFICATIVOS}

\begin{tabular}{|c|c|c|c|c|c|c|}
\hline Probit regre: & -506.7021 & & & & $\begin{array}{l}\text { of obs } \\
2(7) \\
\text { chi2 } \\
\text { R2 }\end{array}$ & $\begin{array}{rr}= & 1094 \\
= & 366.96 \\
= & 0.0000 \\
= & 0.2658\end{array}$ \\
\hline Participa & Coef. & Std. Err. & 2 & $p>|z|$ & {$[95800$} & f. Interval] \\
\hline IJ & -.0015887 & .0004509 & -3.52 & 0.000 & -.002472 & -.000705 \\
\hline 5] & -.2576033 & .0635116 & -4.06 & 0.000 & -.382083 & -1331229 \\
\hline ES & .0111987 & .0032778 & 3.42 & 0.001 & .004774 & (017623. \\
\hline MI & .4450994 & .1049648 & 4.24 & 0.000 & .239372 & 6508267 \\
\hline ST & .8637834 & .1006943 & 8.58 & 0.000 & .666426 & 1.061141 \\
\hline If & .004676 & .000347 & 13.47 & 0.000 & .003995 & .0053562 \\
\hline TF & -.0653669 & .0255369 & -2.56 & 0.010 & -115418 & -0153156 \\
\hline _cons & -1.091211 & . 1932345 & -5.65 & 0.000 & -1.46994 & -.7124778 \\
\hline
\end{tabular}

\section{FUENTE: Elaborado por los autores}

En la decisión de laborar del trabajador secundario tiene efecto positivo la edad del trabajador secundario (ES), la presencia de menores de 5 años en el hogar (M1), trabajador secundario sea hombre (ST), ingresos familiares (IF). Tienen efecto negativo el ingreso del jefe del hogar (IJ), jefe del hogar mujer (SJ) y tamaño de la familia (TF).

El efecto de la edad del trabajador secundario se explica debido a que a medida aumenta la edad del trabajador, podría sentirse cada vez más en una carga familiar. Asimismo, la presencia de menores de 5 años justifica esta decisión, puesto que por la naturaleza humana sabemos que debemos atender las necesidades de nuestros hijos o hermanos menores. Esto se hace evidente en cuanto el trabajador secundario es hombre, que en muchos casos podrían ser los hijos mayores quienes tienen que ver por lo menores. Un resultado no esperado es el hecho que es más probable que decidan laborar los trabajadores secundarios en los cuales los ingresos familiares son mayores, una razón que explicaría este fenómeno es el hábito de trabajar de los trabajadores secundarios ya adquirido.

Por el contrario, los jefes del hogar con mayores ingresos se opondrán a que su cónyuge o hijos formen parte de la masa laboral. Asimismo, si el jefe del hogar es una mujer, muy probablemente es porque su cónyuge no está en condiciones de laborar, o simplemente lo ha abandonado, siendo la mujer la que enfrente los problemas económicos. Más bien llama la atención el efecto negativo del tamaño de la familia en la decisión de trabajar de este trabajador, por cuanto siendo mayor el número menos posibilidades tiene de formar el mercado laboral, una explicación que podría dársele es que corresponda a hogares con hijos pequeños y el trabajador secundario que alcanzó la edad mínima de la PEA tenga como responsabilidad cuidarlos.

Los trabajados referidos no explican las razones por las que los factores significativos tienen una influencia positiva o negativa sobre la decisión de laborar del trabajador secundario, limitándose solo a establecer la dirección, positiva o negativa, de la influencia.

Paz ${ }^{1}$ mostró que la mayor educación del trabajador secundario, el ser varón, el ser hijo del jefe y el tener un salario horario potencial más alto influyen positivamente en esta decisión; en cambio, el ser esposa del jefe del hogar y, siendo la esposa, el tener menores de edad a cargo, el ser hijo del jefe y estar estudiando (a la vez), el tener mayores ingresos de fuentes no laborales y el vivir en un hogar regido por jefe y con nivel educativo alto tienen relación inversa con tal decisión. Aún cuando pueda existir diferencia en la estimación de los coeficientes empleando el modelo logit en comparación con el modelo probit utilizado en el presente trabajo, existe una relación directa entre los coeficientes entre uno y otro modelo, que no altera la significancia de los mismos. Entonces, hay coincidencia en el efecto positivo para la decisión de laborar el que el trabajador secundario sea hombre, y efectos no concordantes la presencia de menores de 5 años en el hogar, aunque la diferencia puede deberse a que en nuestro trabajo no se considera que simultáneamente sea la esposa quien los tiene a su cargo.

La muestra estuvo conformada por 18466 trabajadores en 1992, por 26068 en 1995 y 22230 en 1999, cifras importantes para el análisis de la bondad de los modelos estimados.

La comparación con los estudios realizados por el Ministerio de Trabajo y Promoción del Empleo en Perú ${ }^{2,3}$ es más limitada. En el primero de los trabajos se indica que se empleó un modelo tipo logit, que en nuestra opinión por los resultados mostrados corresponde al modelo lineal de probabilidad, y en el segundo caso aún cuando se empleó el modelo logit, no se presentan los coeficientes de regresión de las variables en estudio, únicamente se muestran el signo de los coeficientes significativos. El primer estudio reveló como factores que favorecen la decisión de trabajar de los trabajadores secundarios su educación y 
edad, así como PET femenina/PET familiar y el número de menores de 14 años/PEA ocupada familiar. En cambio, los factores con efecto negativos fueron el sexo del trabajador secundario, el ingreso laboral del resto de la familia, ingreso no laboral familiar, el número de miembros del hogar y la educación promedio de la PEA.

El segundo estudio muestra que tienen efecto positivo en la decisión del trabajador secundario los años de estudio, la presencia de menores de 5 años en el hogar y de menores de 5 a 13 años a cargo de la cónyuge, la tasa de desocupación familiar, la PET femenina/PET total, el promedio de los años de estudio de la PEA familiar y el tamaño de la familiar. Asimismo, tienen efecto negativo el que el trabajador secundario sea mujer, sea la cónyuge, un hijo que estudia o que la cónyuge tenga menores de 5 años a su cargo, el ingreso del resto de la familia, los ingresos no laborales, los años de estudio del jefe, el tamaño de la familia y los menores de 14/PEA ocupada. Si bien este trabajo es ambicioso en cuanto al número de variables independientes incluidas, no es muy explícito a los argumentos teóricos. La cantidad de variables significativas y la forma de crear estas variables contrasta en cierto modo con el coeficiente R-cuadrado de McFadden, cuyo valor es de 0.2078 , que en cierto modo puede ser indicador de multicolinealidad, menor que el obtenido en nuestro trabajo con un Pseudo $\mathrm{R}^{2}$ de 0.2658 y que los obtenidos por $\mathrm{Paz}^{1}$ con valores de $0.245,0.248$ y 0.249 . Aunque tiene a su favor el empleo de 18309 trabajadores secundarios en la estimación del modelo.

Por su parte Abanto $^{4}$, encontró influencia positiva de la edad del trabajador secundario, el ingreso no laboral de la familia, el ser hijo y los años de estudio del trabajador secundario. Por el contrario, tienen efecto negativo el que el jefe de familia se encuentre ocupado (con cualesquier nivel de ingresos) y su educación, el tamaño de la familia, y que el trabajador secundario estudie, su edad $^{2}$, sea la cónyuge o tenga hijos menores de 5 años o sea un hijo que se encuentra estudiando. Al comparar con nuestros resultados, hay coincidencia del efecto de la edad, no se examinó el efecto cuadrático de la misma, y también respecto al ingreso del jefe del hogar, visto aquí en combinación con la ocupación del mismo. Aunque en este trabajo no se presenta el pseudo valor de $\mathrm{R}^{2}$, a diferencia del trabajo del Ministerio de Trabajo y Promoción del Empleo en Perú ${ }^{3}$ que utilizó información 18309 de trabajadores secundarios, ahora se empleó 5573809 sin construir variables muy elaboradas, en ambos casos basados en ENAHO 2001III.

El efecto de la contribución marginal en la decisión de laborar del trabajador secundario se muestra en la tabla 2. Las variables con efecto positivo con mayor influencia sobre la decisión de laborar del trabajador secundario es que sea hombre y haya la presencia de menores de 5 años en el hogar. En cambio, se oponen más a esta decisión que el jefe del hogar sea mujer.
TABLA N ${ }^{\circ} 2$

EFECTOS MARGINALES DE LA FUNCIÓN PROBIT DE LA

PARTICIPACIÓN LABORAL DEL TRABATADOR SECUNDARIO

Probit regression, reporting marginal effects

Log likelihood $=-506.70216$

Number of obs $=1094$

LR Chi2 $(7)=366.96$

Prob $>$ chi2 $=0.0000$

Pseudo R2 $=0.2658$

\begin{tabular}{|c|c|c|c|c|c|c|}
\hline Partic d & $d F / d x$ & std. Err. & 2 & $p>|z|$ & $x$-bar & 958 C.I. \\
\hline IJ & -.0005425 & .0001533 & -3.52 & 0.000 & 150.904 & $-.000843-.0002$ \\
\hline 5] & -.0879661 & .0217736 & -4.06 & 0.000 & .83638 & $-.130642-.045291$ \\
\hline ES & .0038241 & .0011201 & 3.42 & 0.001 & 32.1874 & 006019. \\
\hline$M M^{*}$ & 年. 1584676 & .038423 & 4.24 & 0.000 & .287934 & .08316. 233775 \\
\hline $5 T^{*}$ & . 3059131 & .0356323 & 8.58 & 0.000 & . 35649 & .236075 \\
\hline IF & 等. 0015967. & .0001204 & 13.47 & 0.000 & 105.695 & 001361. 001833 \\
\hline TF & -.0223214 & . 0087167 & -2.56 & 0.010 & 4.62157 & $-.039406-005237$ \\
\hline
\end{tabular}

FUENTE: Elaborado por los autores.

\section{- INTENSIDAD DE PARTICIPACIÓN EN MERCADO LABORAL}

El modelo de regresión tobit para examinar los factores asociados al número de horas que el trabajador secundario labora a la semana se muestra en la tabla 3. En forma similar, se presenta el modelo estimado después de haber eliminado los factores no significativos.

Los factores asociados a la intensidad laboral de los trabajadores secundarios son los mismos que para la decisión de laborar. La influencia de estos factores se da en la misma dirección. Es decir, los factores que determinan una mayor intensidad laboral en los trabajadores secundarios son la edad del trabajador secundario (ES), la presencia de menores de 5 años en el hogar (M1), trabajador secundario mujer (ST), ingresos familiares (IF), y los factores asociados a una menor intensidad laboral son el ingreso del jefe del hogar (IJ), jefe del hogar mujer (SJ) y tamaño de la familia (TF).

Los trabajos realizados por el Ministerio de Trabajo y Promoción del Empleo ${ }^{2,3}$ no son aquí discutidos debido a que analizan la tasa de participación de la familia en la PEA, y no la intensidad laboral definida como el número de horas que el trabajador secundario labora a la semana.

En forma similar al presente trabajo, en el estudio de $\mathrm{Paz}^{1}$, se encontró en cada uno de los periodos estudiados, que las variables asociadas a la decisión del trabajador secundario de laborar son las mismas que influyen en el número de horas semanales que dedican a laborar, con influencia en la misma dirección, la cual se mantiene a lo largo de los tres periodos estudiados: 1992, 1995 y 1999. La educación del jefe del hogar es la única variable que no mostró influencia significativa el 1999. 
TABLA ${ }^{\circ} 3$

ANÁLISIS TOBIT DEL TIEMPO QUE LABORA SEMANALMENTE

EL TRABAJADOR SECUNDARIO RETIRANDO LOS FACTORES

NO SIGNIFICATIVOS

. tobit Horas IJ SJ ES MI ST IF TF, 11

Tobit regression

Log likelihood $=-2174.2623$

Number of obs $=1094$
LR chi2 $(7)=349.75$
Prob $>$ chi2 $=0.0000$
Pse
Pseudo R2 $\approx 0.074$

\begin{tabular}{r|rrrrrr}
\hline Horas & Coef. & std. Err. & $\mathrm{t}$ & $\mathrm{p}\rangle|\mathrm{t}|$ & \multicolumn{2}{c}{ [95\% conf. Interva1] } \\
\hline IJ & -.0780256 & .0179088 & -4.36 & 0.000 & -.1131654 & -.0428859 \\
SJ & -8.2722095 & 2.737056 & -3.02 & 0.003 & -13.64261 & -2.901584 \\
ES & .4145893 & .1345696 & 3.08 & 0.002 & .1505438 & .6786348 \\
M1 & 18.27308 & 4.211612 & 4.34 & 0.000 & 10.00927 & 26.53689 \\
ST & 34.41692 & 4.025222 & 8.55 & 0.000 & 26.51884 & 42.31501 \\
IF & .1721762 & .0133773 & 12.87 & 0.000 & .1459279 & .1984245 \\
TF & -2.595527 & 1.073869 & -2.42 & 0.016 & -4.702617 & -.4884371 \\
Cons & -41.09662 & 8.21827 & -5.00 & 0.000 & -57.222209 & -24.97115 \\
\hline /sigma & 46.82082 & 2.022762 & & & 42.85185 & 50.78978 \\
\hline
\end{tabular}

$\begin{array}{ll}\text { Obs. summary: } & 739 \text { left-censored observations at Horas }<=0 \\ & 355 \text { uncensored observations } \\ & 0 \text { right-censored observations }\end{array}$

FUENIE: Elaborado por los autores.

La coincidencia de los factores asociados significativos para cada uno de las variables de la participación laboral de los trabajadores secundarios, no se confirma en el estudio realizado por Abanto ${ }^{4}$. En primer lugar, el ingreso del trabajador que no fue incluida en el modelo probit, ahora tiene efecto significativo en el número de horas semanales que labora, lo cual refleja el costo de oportunidad, de manera que mientras mayor es el ingreso que percibe se está dispuesto a trabajar más. Asimismo, la variables que cambiaron el sentido de su influencia fueron el tamaño de la familia que ahora muestra un efecto positivo contrario a los resultados de muestro trabajo, y los años de estudio del trabajador secundario.

Finalmente, se debe mencionar que aún cuando la muestra considerada en el presente estudio es suficientemente grande, es inferior a las utilizadas en los trabajos referidos, aspecto que como se indicó tiene efecto sobre la bondad de las estimaciones en caso de que el estudio se hubiera fraccionado en grupos específicos de trabajadores secundarios, como en algunos de los trabajos mostrados.

\section{CONCLUSIONES}

Los factores asociados positivamente a la participación laboral de los trabajadores secundarios en el distrito de Guadalupe, definido tanto en la decisión de participar en la fuerza laboral como en el número de horas semanales laboradas, fueron la edad del trabajador secundario, la presencia de menores de 5 años en el hogar, trabajador secundario mujer, ingresos familiares. En cambio, los factores asociados negativamente fueron el ingreso del hogar, jefe del hogar mujer y el tamaño de la familia.

\section{AGRADECIMIENTOS}

A la Universidad Nacional de Trujillo, la mejor Universidad del Norte del Perú, por hacer posible el presente estudio.

\section{REFERENCIAS BIBLIOGRÁFICAS}

[1]. Paz J. Oferta laboral de reserva en la Argentina (una nueva mirada sobre el efecto del trabajador adicional. Universidad Nacional de Salta; 2001.

[2]. Ministerio de Trabajo y Promoción del Empleo. Programa de estadísticas y estudios laborales. Participación laboral: un enfoque de oferta familiar de trabajo. Boletín de economía laboral; 2000:16.

[3]. Ministerio de Trabajo y Promoción del Empleo. Programa de estadísticas y estudios laborales. El trabajador independiente urbano: un enfoque de oferta laboral familiar. Boletín de economía laboral; 2002:22-24; 43-66.

[4]. Abanto E. Modelos probit y tobit aplicados al estudio de la oferta laboral de los trabajadores secundarios [monografía para título profesional]. Lima: Universidad Nacional Mayor de San Marcos; 2003.

[5]. Uribe J. Ensayos de economía aplicada al mercado laboral. Colombia: Programa Editorial Universidad del Valle; 2006.

E-mail: cminchonm@gmail.com carlos.sanchez.t@gmail.com 